\title{
Staphylococcus enterotoxin B-induced T cells can efficaciously protect against type 1 diabetes in non-obese diabetic mice
}

\author{
YELEI GUO ${ }^{I}$, LEI XIA ${ }^{2}$, SHILUN ZHANG ${ }^{3}$, JIANG ZHONG ${ }^{3}$, YU CHEN ${ }^{1}$ \\ ${ }^{1}$ Department of Immunology Institute of Basic Medicine, School of Life Sciences, Chinese PLA General Hospital, Beijing, 100853, China \\ ${ }^{2}$ Department of Medical Administration, Chinese PLA General Hospital, Beijing, 100853, China \\ ${ }^{3}$ Department of Microbiology and Microbial Biotechnology, School of Life Sciences, Fudan University, Shaghai, 200433, China \\ Yelei Guo and Lei Xia contributed equally to this work.
}

\begin{abstract}
Type 1 diabetes (TID), an autoimmune disease, can be protected against by natural killer T (NKT) cells. Several attempts demonstrate that NKT cells also can be produced by inducing with Staphylococcus enterotoxin $B(S E B)$ in addition to its classical activated antigen $\alpha$-galactosylceramide. Here, we examined a potential usage of SEB-induced T (SEB-T) cells for the treatment of TID. We established the immunophenotypes of SEB-T cells via flow cytometry, and in consequence, enriched in $C D 8^{+} N K T$ cells after SEB stimulated. A high level of transforming growth factor $\beta$ (TGF- $\beta$ ), detected by RT-PCR and ELISA, was first observed to be expressed and secreted by these SEB-T cells. Mixed lymphocyte reactions indicated that SEB-T cells could not produce a response to mitogens and allogeneic lymphocyte, and can inhibit lymphocytes response to mitogens. In an animal model, our data indicated that infusion of SEB-T cells in non-obese diabetic mice was well tolerated and could ameliorate hyperglycemia and maintain the blood glucose nearly on normal level until sacrifice. Strikingly, infusion of SEB-T cells resulted in an increase in the serum TGF- $\beta$ level. These data raise the possibility that SEB-T cells can protect against T1D, which is associated with NKT cells generated in these SEB-induced cells.
\end{abstract}

Key words: immunomodulatory, type 1 diabetes, Staphylococcus enterotoxin B, CD8+ natural killer T, transforming growth factor- $\beta$, adoptive transfer.

(Cent Eur J Immunol 2015; 40 (3): 292-299)

\section{Introduction}

Type 1 diabetes (T1D) is an autoimmune disease that is characterized by the destruction of pancreatic islet $\beta$ cells through the action of autoimmune T helper (Th) 1 cells, $\mathrm{CD}^{+} \mathrm{T}$ cells and macrophages $[1,2]$. Although current traditional therapeutic approaches such as insulin administration provide beneficial protection for T1D, they are not very effective in delaying the loss of functional $\beta$ cells [3]. Therefore, strategies to ameliorate the hyperglycemic symptoms and simultaneously suppress abnormal immune activity would provide future therapeutic options for T1D.

Importantly, numerous studies have highlighted that natural killer T (NKT) cells have immunomodulatory ability that impedes the onset of T1D in mice $[4,5]$. Natural killer $\mathrm{T}$ cells were first identified as the fourth type of lymphocytes in the co-expressed cell surface markers of both $\mathrm{T}$ cells (such as $\alpha / \beta$ TCR) and NK cells (CD161), which mediate protective immune responses against tumor infections, transplant rejection and autoimmune diseases [6, 7].
Recent studies have indicated that NKT cells can be effective for protecting against T1D via modulation following that NKT cells induce the differentiation of dendritic cells (DCs) to the anergy state and generate Th2 and Tregs responses $[8,9]$. In addition to $\alpha$-galactosylceramide, the classical antigen activating NKT cells, lipid or peptide antigens such as anti-CD3 F(ab') ${ }_{2}$ have also been found to activate NKT cells [10-12]. A few reports have demonstrated that NKT cells also can be induced by Staphylococcus enterotoxin B (SEB) [11]. However, the therapeutic effects of NKT cells activated by SEB in terms of protection against T1D have rarely been reported.

Therefore, we evaluated the biological characteristics of SEB-stimulated T (SEB-T) cells by developing a protocol for their effective expansion in vitro to establish their ameliorating hyperglycemia potential in T1D. To investigate the possible therapeutic effects involved in SEB-T cell infusion, we took advantage of non-obese diabetic (NOD) mice, which is a human T1D animal model and

Correspondence: Prof. Yu Chen, PhD, Chinese PLA General Hospital, e-mail: guoyl1986@ foxmail.com; Prof. Jiang Ahong, PhD, Fudan University, e-mail: jzhong@ fudan.edu.cn 
significantly characterized by the decrease in the numbers or function of tissue resident NKT cells, regulatory T cells (Tregs), DCs, and macrophages [13, 14], where $70-80 \%$ of the female mice spontaneously develop the disease [15]. We performed SEB-T cell infusion after the appearance of hyperglycemia and then measured the effects on protection against T1D in NOD mice.

\section{Material and methods}

\section{Animals}

Forty female C57BL/6 mice, twenty female BALB/c mice and twenty-six female and 5-week-old NOD mice were obtained from the Chinese Academy of Medical Sciences and bred in a specified-pathogens free room at the Chinese People's Liberation Army General Hospital Animal Experiment Center. All animal experiments were approved by the Institutional Animal Care and Use Committee at the Chinese General PLA Hospital.
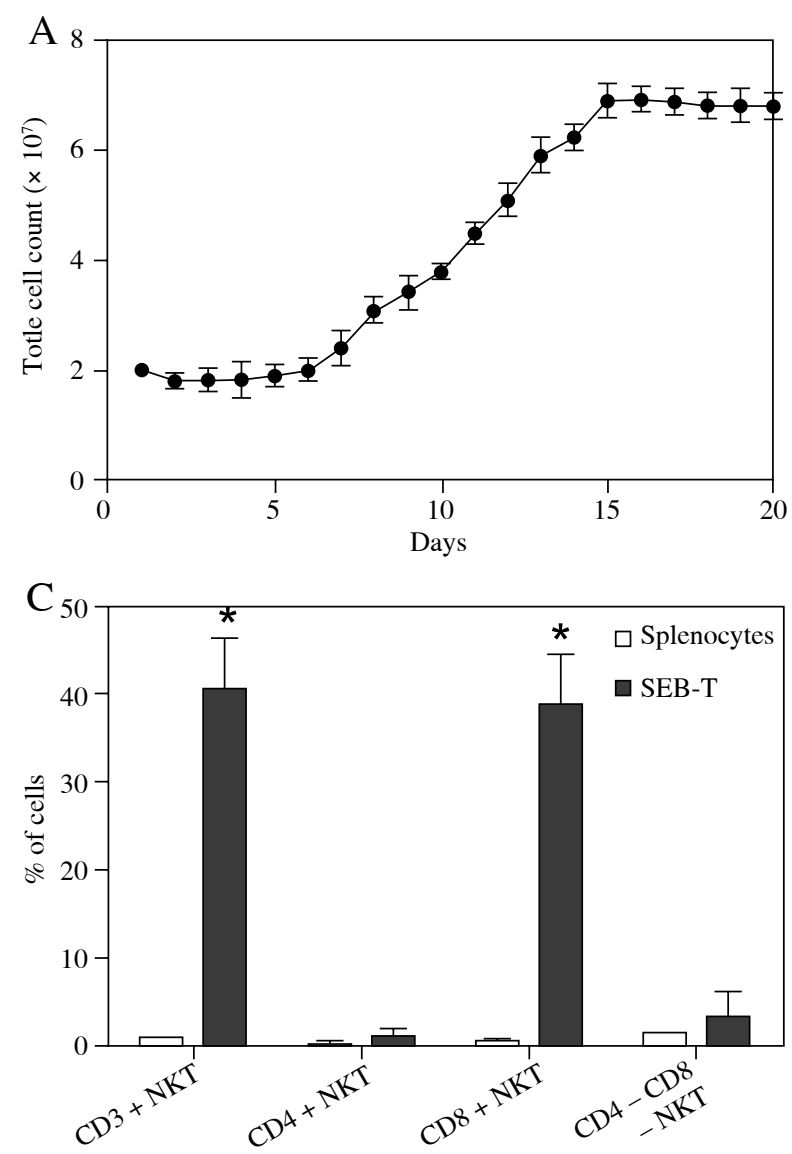

\section{Generation of SEB-T cells}

$5 \times 10^{6}$ cells $/ \mathrm{ml}$ splenocytes, from C57BL/6 mice, were cultured with $200 \mathrm{ng} / \mathrm{ml} \mathrm{SEB}$ (Sigma, USA) at $37^{\circ} \mathrm{C}$ with $5 \% \mathrm{CO}_{2}$ incubator. A half volume of culture medium was replaced with fresh RPMI 1640 medium (Gibco, USA, supplemented with $10 \%$ FBS, 25 mmol/l HEPES, 4 mmol/l L-glutamine, $100 \mathrm{U} / \mathrm{ml}$ penicillin, $100 \mu \mathrm{g} / \mathrm{ml}$ streptomycin) every 5 days. Staphylococcus enterotoxin B-stimulated $\mathrm{T}$ cells were harvested after 15 days.

\section{Phenotype of SEB-T cells}

Staphylococcus enterotoxin B-stimulated T cells were collected and stained with FITC-conjugated anti-CD69, PerCP-conjugated anti-CD3, FITC-conjugated anti-CD4, PE-conjugated anti-CD8, and APC-conjugated anti-NK1.1 antibodies (BD biosciences, USA). Data analysis was performed by FACSCalibur flow cytometer (BD biosciences, USA). Normal splenocytes from C57BL/6 were used as controls.
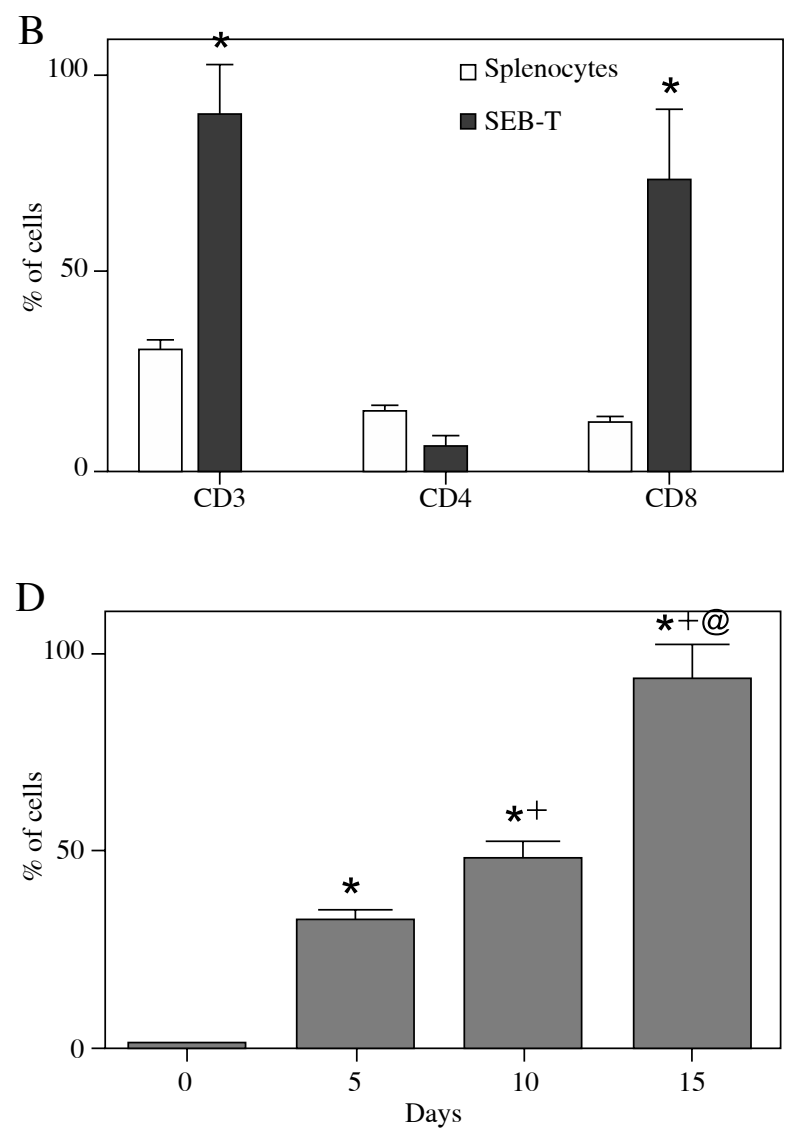

Fig. 1. Identification of SEB-T cells characteristics. A) Cellular proliferation of SEB-T cells: cells were counted every day using trypan blue after co-culturing C57BL/6 mouse splenocytes with SEB. B, C) Phenotypic analysis of SEB-T cells. All cells were stained with CD3-PerCP, CD4-FITC, CD8-PE and NK1.1-APC antibodies $\left({ }^{*} p<0.01\right)$. D) The percentage of CD69-positive cells in the cell population $\left({ }^{*} p<0.01\right.$ vs. day $0,{ }^{+} p<0.01$, vs. day $5,{ }^{\circledR} p<0.01$, vs. day 10$)$ 
Table 1. Primer sequences and size of expected products

\begin{tabular}{cccc}
\hline Gene & Upstream primer (5'-3') & Downstream primer (5'-3') & $\begin{array}{c}\text { Expected } \\
\text { size(bp) }\end{array}$ \\
\hline IL-2 & -AAGCTCTACAGCGGAAGCAC- & -GTCCACCACAGTTGCTGACT- & 370 \\
IL-4 & -ATGGGTCTCAACCCCCAGCTAGT- & -GCTCTTTAGGCTTTCCAGGAAGTC- & 398 \\
IL-5 & -TCCCTGCTACTCTCCCCAAAC- & -TGGCACAGTCTGATTCATACATAGG- & 78 \\
IL-6 & -TTCCATCCAGTTGCCTTCTTGG- & -CTTCATGTACTCCAGGTAG- & 360 \\
IL-10 & -GGTTACTTGGGTTGCCAAGC- & -TTCGGAGAGAGGTACAAACG- & 418 \\
IFN- $\gamma$ & -AACGCTACACACTGCATCTTGG- & -GACTTCAAAGAGTCTGAGG- & 237 \\
TGF- $\beta$ & -TATAGCAACAATTCCTGGCGT- & -TCCTAAAGTCAATGTACAGCT- & 390 \\
FoxP3 & -CCCAGGAAAGACAGCAACCTT- & -TTCTCACAACCAGGCCACTTG- & 88 \\
$\beta$-actin & -TGGAATCCTGTGGCATCCATGAAAC- & -TAAAACGCAGCTCAGTAACAGTCCG- & 348 \\
\hline
\end{tabular}

\section{Reverse transcription-polymerase chain reaction}

Staphylococcus enterotoxin B-stimulated T cells were used to extract total cellular RNA. cDNAs were obtained using M-MuLV reverse transcriptase (Fermentas, USA) and oligo dT primer, following the manufacturer's protocol. PCRs were conducted with $0.5 \mu \mathrm{l}$ of cDNA as the template and the primer pairs listed in Table 1. The PCR was performed as described previously [16].

\section{Enzyme-linked immunosorbent assay}

The cell culturing supernatants were collected on day 15 and analyzed for the secretion of interleukin (IL)-2, IL-4, IL-5, IL-6, IL-10, interferon $\gamma$ (IFN- $\gamma$ ) and TGF- $\beta$ using ELISA kits (BD Pharmingen, San Jose, CA, USA).

\section{Mixed lymphocytes reaction}

Mixed lymphocytes reaction, included (1) $5 \times 10^{5}$ SEB-T cells were separately cultured with $5 \mu \mathrm{g} / \mathrm{ml}$ ConA, LPS, $5 \times 10^{5} \mathrm{C} 57 \mathrm{BL} / 6$ and BALB/c mouse splenocytes that were pre-treated with $20 \mu \mathrm{g} / \mathrm{ml}$ mitomycin. (2) SEB-T cells were pre-treated with $20 \mu \mathrm{g} / \mathrm{ml}$ mitomycin and $5 \times$ $10^{5}$ cells cultured with the same number of C57BL/6 or BALB/c mouse splenocytes, (3) $5 \times 10^{5}$ C57BL/6 mouse splenocytes separately cultured with $5 \mu \mathrm{g} / \mathrm{ml}$ ConA and LPS, then, $5 \times 10^{5}$ cells SEB-T cells were added, were all performed in 96 -well plates and incubated at $37^{\circ} \mathrm{C}$ in $5 \%$ $\mathrm{CO}_{2}$ for 72 hours. MTT assay was conducted to determine cell viability as follows. Data acquisition was performed with a microplate reader (BIO-RAD, USA).

\section{Animal experiments}

The incidence of T1D in NOD mice is defined as two consecutive fasting blood glucose measurements $\geq 11.6$ $\mathrm{mmol} / \mathrm{l}$. Twenty-six NOD mice were divided into three groups. NOD mice, within 7 days of the onset of overt diabetes, were intravenously infused with $5 \times 10^{6} \mathrm{SEB}-\mathrm{T}$ cells weekly for 2 consecutive weeks (SEB-T group, $n=10$ ). C57BL/6 mice splenocytes (splenocytes group, $n=8$ ) and PBS (vehicle group, $n=8$ ) were also used to treat the NOD mice. The level of blood glucose was measured throughout the experiments with a glucometer-ACCU-CHEK Advantage Meter (Roche Diagnostics GmbH, Mannheim, Germany) weekly. At 30 weeks, all NOD mice were sacrificed. Blood sera were collected, and serum cytokines IL-2, IL-4, IL-5, IL-6, IL-10, IFN- $\gamma$ and TGF- $\beta$ were analyzed using ELISA kits (BD Pharmingen, San Jose, CA, USA).

\section{Statistical analysis}

The results are presented as the mean \pm standard error of the mean of triplicate determinants. GraphPad Prism version 5.0 was used for the statistical analysis of the data. Two-way analysis of variance was used to determine the significance of the differences between the means in all experiments. A $p$ value of less than 0.05 was considered to be statistically significant.

\section{Results}

\section{General characteristics of SEB-T cells}

First, we established a stable system for the proliferation of SEB-T cells in vitro, which were generated from C57BL/6 mice splenocytes. After culturing for 15 days, the total number of these cells reached the peak of cell proliferation and was increased by 3.46 -fold. Then, the cellular proliferation entered the plateau phase (Fig. 1A). The immunophenotypes of the SEB-T cells were tested by FACS. The population of these cells mainly contained $90.2 \%$ of $\mathrm{CD}^{+} \mathrm{T}$ cells, $73.46 \%$ of $\mathrm{CD}^{+} \mathrm{T}$ cells and $40.21 \%$ of $\mathrm{CD}^{+} \mathrm{NKT}$ cells $\left(\mathrm{CD}^{+}{ }^{+} \mathrm{NK} 1.1^{+}\right)$that included approximately $38.58 \%$ of $\mathrm{CD} 8^{+} \mathrm{NKT}$ cells $\left(\mathrm{CD} 8^{+} \mathrm{NK} 1.1^{+}\right)$ and very few $\mathrm{CD}^{+}{ }^{+} \mathrm{NKT}$ and $\mathrm{CD}^{-}{ }^{-} \mathrm{CD} 8^{-} \mathrm{NKT}$ cells (Fig. 1B, C). The level of activation marker CD69 on SEB-T cells was significantly increased, which achieved $83.7 \%$ after 15 days of culturing (Fig. 1D).

mRNA of IL-2, IL-4, IL-5, IL-6, IL-10, TGF- $\beta$, IFN- $\gamma$ and FoxP3 were tested by RT-PCR for SEB-T cells. The level of mRNA TGF- $\beta$ and IFN- $\gamma$ for SEB-T cells were evidently increased (Fig. 2A). In addition, we examined the secretion of cytokines IL-2, IL-4, IL-5, IL-6, IL-10, TGF- $\beta$ and IFN- $\gamma$ by ELISA. The level of TGF- $\beta$ for these 


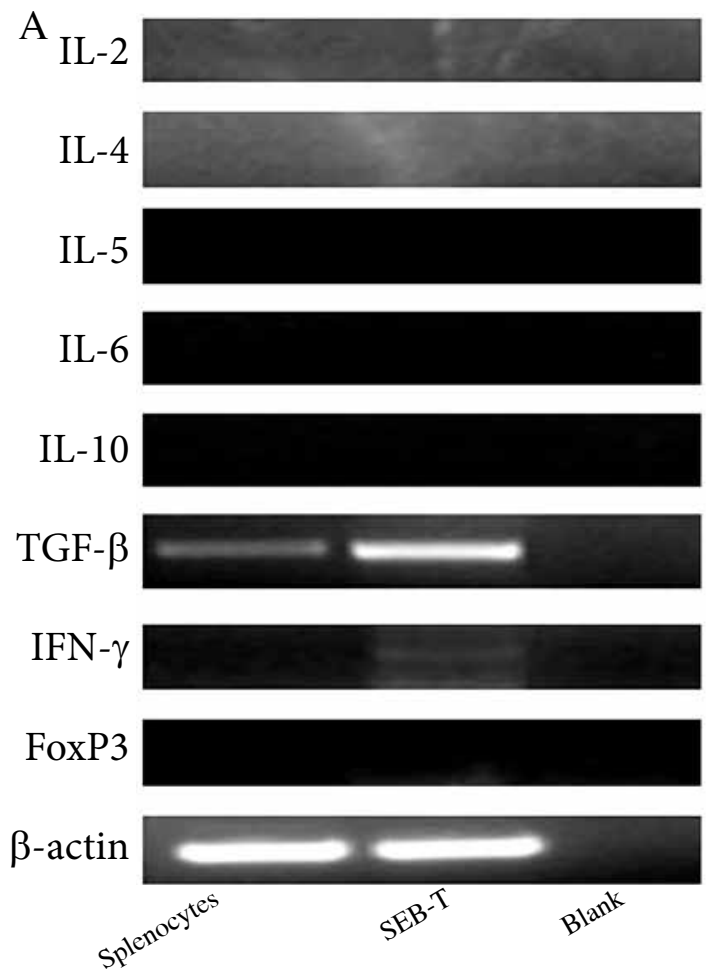

B

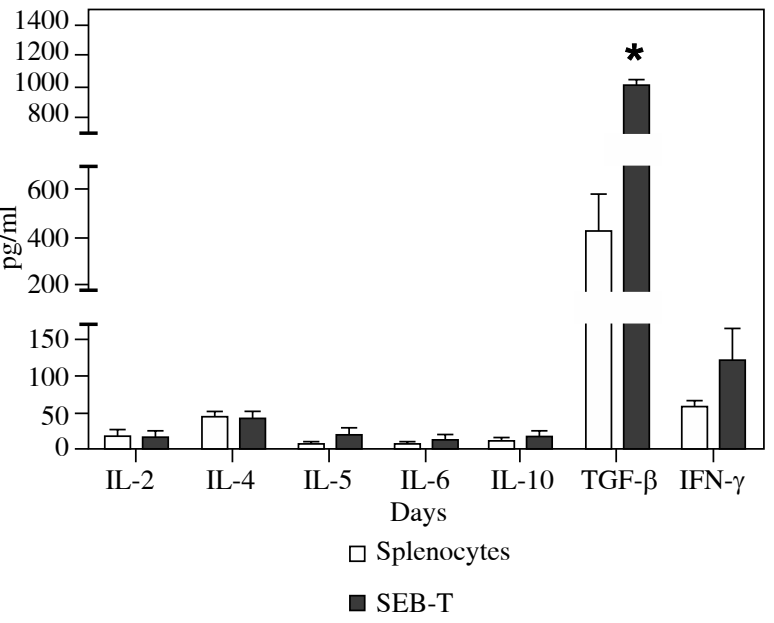

Fig. 2. Analysis of the expression of cytokines in the SEB-T cells. A) Expression of the mRNAs for IL-2, IL-4, IL-5, IL-6, IL-10, TGF- $\beta$, IFN- $\gamma$ and FoxP3 were analyzed by RT-PCR. B) Secretion of cytokines IL-2, IL-4, IL-5, IL-6, IL-10, TGF- $\beta$ and IFN- $\gamma$ were analyzed by ELISA $(* p<0.01)$

cells was significantly increased. Interferon $\gamma$ was also increased, but there were no significant differences noted (Fig. 2B).

\section{Immune response of SEB-T cells in vitro}

Second, we examined the immune response of SEB-T cells by mixed lymphocytes reaction in vitro. SEB-T cells were significantly less responsive to ConA, LPS and autoor allogeneic splenocytes (Fig. 3A). Meanwhile, the immune response for allogeneic $\mathrm{BLAB} / \mathrm{c}$ mouse splenocytes to SEB-T cells was also significantly decreased (Fig. 3B). Interestingly, SEB-T cells could prominently suppress the splenocyte response to ConA and LPS (Fig. 3C).

\section{SEB-T cell infusion ameliorated hyperglycemia in T1D NOD mice}

Finally, we evaluated the therapeutic effect of protection against hyperglycemia in NOD mice by infusion of SEB-T cells. Once hyperglycemic symptoms appeared, NOD mice were immediately treated with SEB-T cells. The mice showed no signs of panic, irritability, weakness, diarrhea, or other symptoms after SEB-T cells were injected through the caudal vein. After infusion with C57BL/6 mice splenocytes, some symptoms, for example, panic, diarrhea, and urinary and fecal incontinence, were observed in the hyperglycemic NOD mice. Preliminary experiments revealed that NOD mice suffered from significant side effects after the injection of allogeneic splenocytes, whereas the injection of SEB-T cells did not result in any toxicity.

In this experiment, NOD mice displayed hyperglycemia at 14 weeks when the concentration of blood glucose was approximately $12.7 \mathrm{mmol} / \mathrm{l}$ (Fig. 4A). The vehicle group showed a persistently high level of blood glucose during the study period. The splenocytes group and SEB-T group were performed at 15 weeks and 16 weeks. Compared with the splenocytes group, the SEB-T cell-treated NOD mice immediately showed a significant decrease in the blood glucose level. The levels decreased to normal and remained approximately normal until the mice were sacrificed at 30 weeks. To evaluate the mechanism of potential protection against hyperglycemic activity of SEB-T cells, we measured the levels of IL-2, IL-4, IL-5, IL-6, IL-10, IFN- $\gamma$ and TGF- $\beta$ in blood sera that were harvested when all the NOD mice were sacrificed at 30 weeks. The levels of TGF- $\beta$ in the SEB-T group were significantly higher than in the splenocytes group (Fig. 4B).

\section{Discussion}

A number of previous studies have demonstrated that NKT cells are capable of protecting against T1D in ani- 

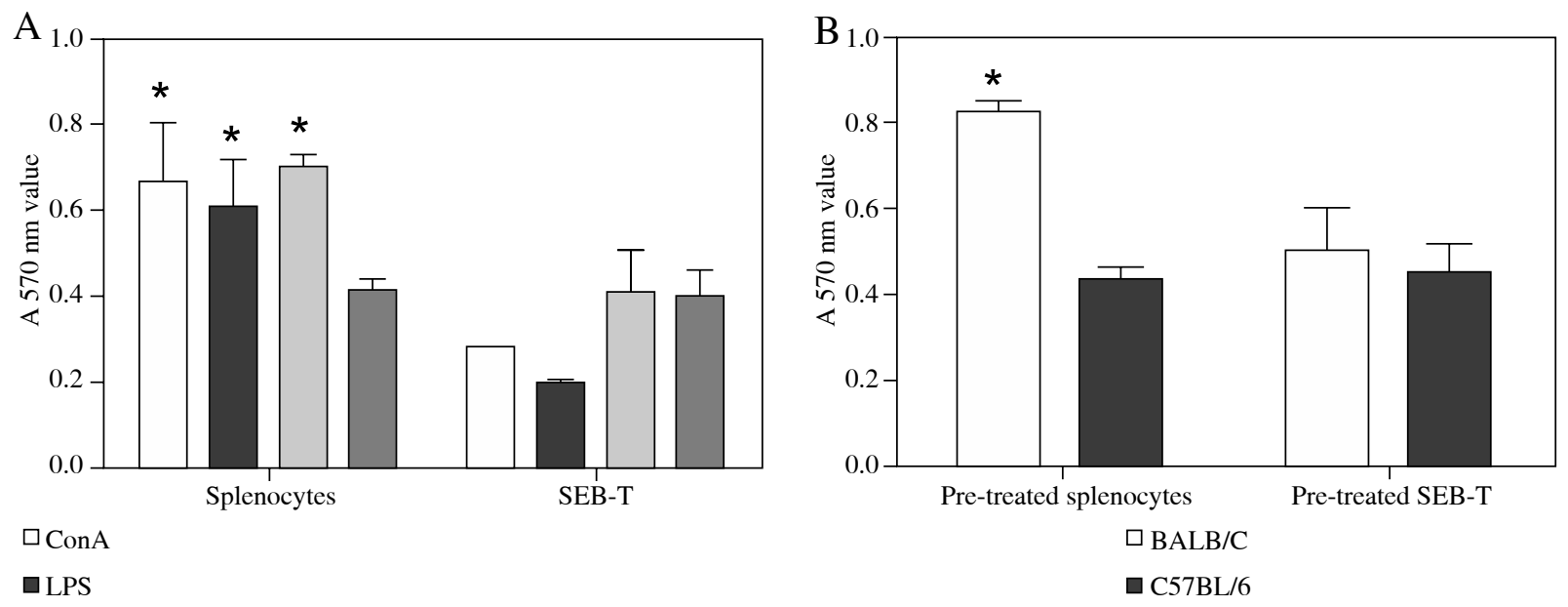

$\square$ Pre-treated BALB/C splenocytes

$\square$ Pre-treated C57BL/6 splenocytes

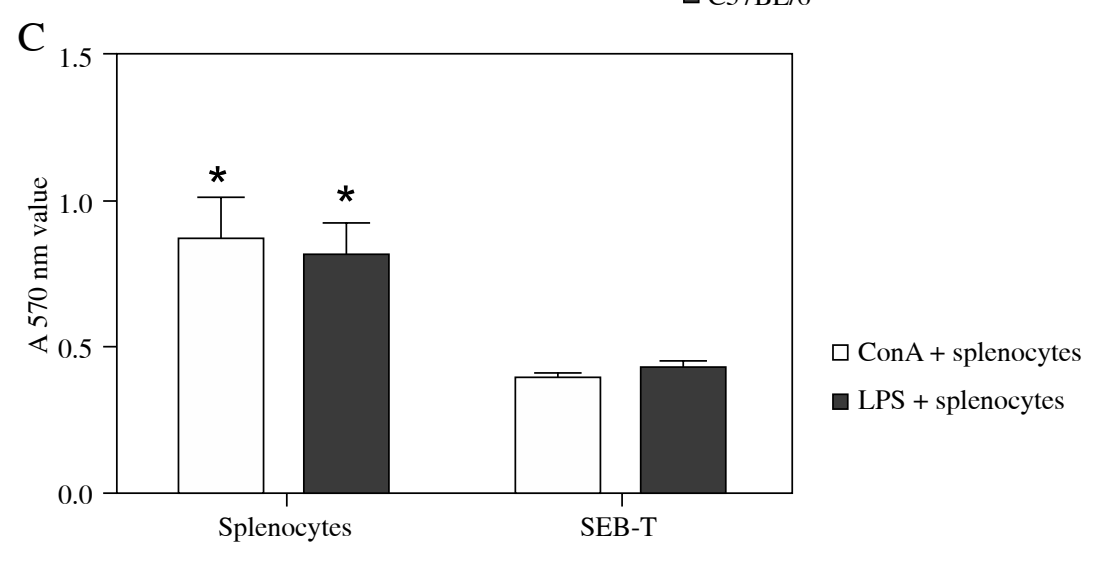

Fig. 3. Immune reaction of SEB-T cells in vitro. A) Immune anergy: C57BL/6 mouse splenocytes and SEB-T cells were cultured in the presence of the stimulus ConA, LPS, pre-treated BALB/C and C57BL/6 splenocytes, respectively. B) Pre-treated C57BL/6 mouse splenocytes and SEB-T cells were co-cultured with normal BALB/C or C57BL/6 splenocytes. C) ConA or LPS were added to splenocytes together with splenocytes or SEB-T cells. The cells of all experiments were cultured for 72 hours. Then, the MTT assay was used to determine the cell viability in the above experiments $(* p<0.01)$

mal models $[12,13]$. Natural killer T cells can be activated by glycolipid or peptide antigens and proliferated to play a role in immune regulation. In recent years, numerous studies have shown that the superantigen SEB also can induce an NKT cell response [11]. Successful attempts on the treatment of autoimmune disease T1D with SEB-activated NKT cells have hardly ever been reported. Therefore, the therapeutic effect of protection against T1D in NOD mice with SEB-T cells was evaluated to explore these outstanding concerns in this study. Consistent with other reports $[12,13]$, our results proved that infusion of SEB-T cells, mainly enriched CD8 ${ }^{+} \mathrm{NKT}$ cells, also could effectively ameliorate hyperglycemic symptoms in T1D NOD mice.

Recent studies have indicated that NKT cells have the ability to ameliorate hyperglycemia and protect against T1D [7, 8]. Theoretically, NKT cells can act as the ideal treatment to control T1D by inducing the differentiation of DCs to the anergy state via the pathways involving cell contacts and Th2 cytokines, such as IL-4, wherein these anergy-state DCs prevent auto-reactive $\mathrm{T}$ cells responses and also generate Th2 and Tregs responses [8, 9]. However, compared with NKT cell therapy for T1D reported in these studies, CD8 ${ }^{+} \mathrm{NKT}$ cells enriched with SEB-T cells had the capacity to therapeutically protect against T1D in NOD mice in the long run possibly through the pathway of significantly increased TGF- $\beta$ secretion. Upon further analysis of the beneficial therapeutic effects in this model, we found that the enrichment of the serum level of cytokine TGF- $\beta$ might provide the ability to ameliorate hyperglycemia and offer continuing protection against the state. Significantly, reversal of T1D by TGF- $\beta$ has been reported in some studies $[17,18]$. When summarizing these studies, it must be realized that several potential reasons have explained the results such as the fact that TGF- $\beta$ has been found (1) to modulate the autoimmune disease [19]; (2) to 

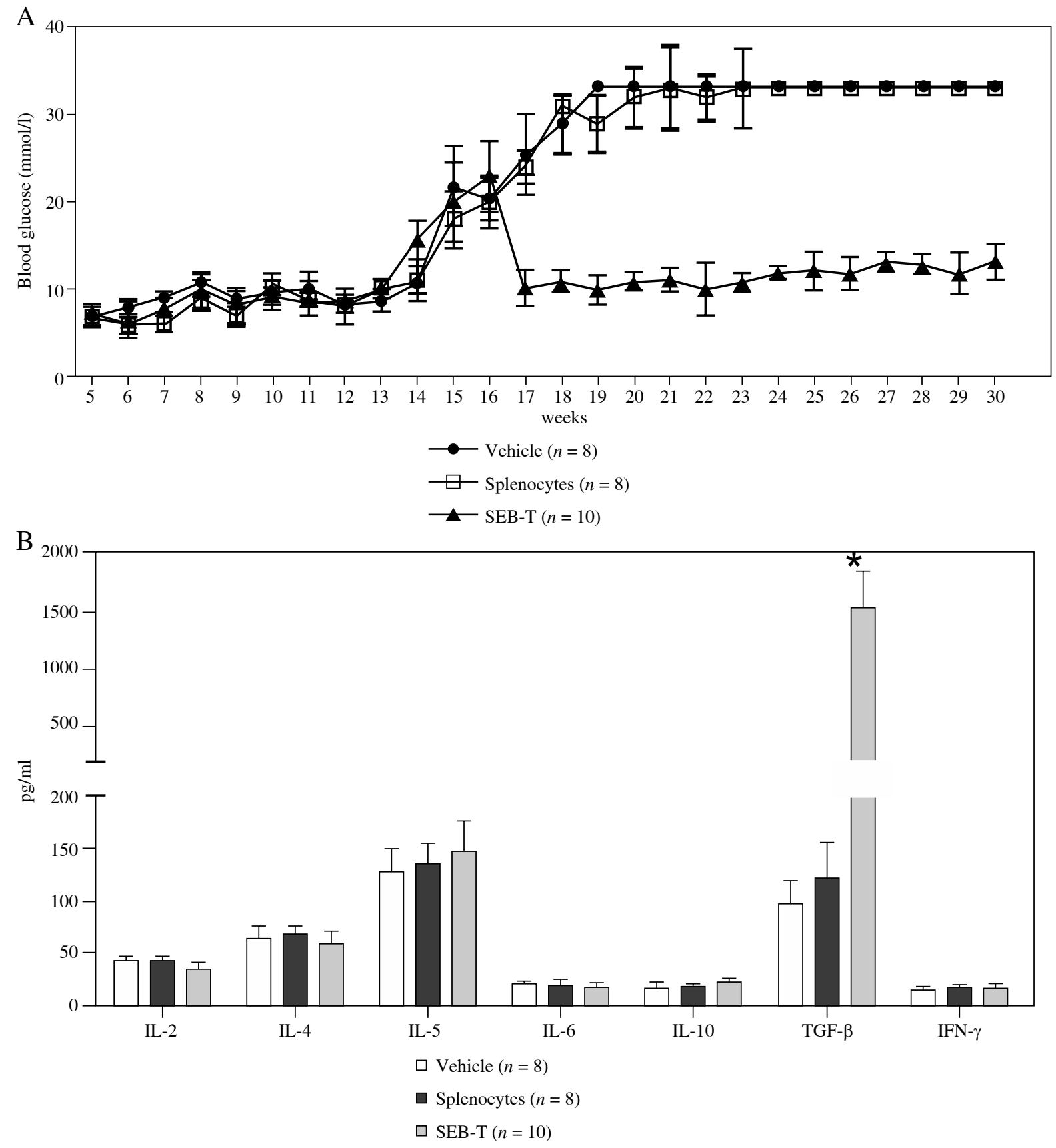

Fig. 4. SEB-T cells were used to treat T1D NOD mice. A) Infusion of SEB-T cells ameliorates hyperglycemia in NOD mice during blood glucose increased. After two consecutive fasting blood glucose measurements $\geq 11.6 \mathrm{mmol} / \mathrm{l}$, diabetic NOD mice received $5 \times 10^{6} \mathrm{SEB}-\mathrm{T}$ cells or C57BL/6 mouse splenocytes in $0.2 \mathrm{ml}$ of physiological saline, respectively. The blood glucose level was determined consecutively in alert, fasted mice using a glucometer-ACCU-CHEK Advantage Meter. B) After NOD mice were sacrificed at 30 weeks, the serum cytokines IL-2, IL-4, IL-5, IL-6, IL-10, TGF- $\beta$ and IFN- $\gamma$ were analyzed by ELISA $(* p<0.01)$

maintain the peripheral tolerance by nadve $\mathrm{CD} 4^{+}$Tregs, and even to induce the differentiation of CD4+ Tregs [20], which play a critical role in the amelioration and protection against T1D [21]; and (3) to derive the tolerance of DCs to the pancreatic islet b cells, as well as to decrease the inflammatory cells included effector and memory CD8 ${ }^{+}$ $\mathrm{T}$ cells [22]. Together, these findings are additional evidence that the infusion of SEB-T cells-enriched CD8 ${ }^{+} \mathrm{NKT}$ 
cells could efficiently ameliorate hyperglycemic symptoms of T1D in NOD mice possibly via TGF- $\beta$ secretion.

Consistent with the notion that immunomodulatory ability is the main characteristic of NKT cells [7], we observed that SEB-T cells also possessed this feature in vitro. According to the results of mixed lymphocyte reaction, SEB-T cells could not respond to mitogens and could inhibit lymphocytes reacting to mitogens. Moreover, it was the most important that these cells could not interact with allogeneic lymphocytes. Therefore, these cells had immune anergy, lower immunogenicity and inhibited the biological function, which could not cause significant side effects like GVHD after infused into allogeneic T1D NOD mice.

At present, many reports indicate that Tregs play a critical role in the autoimmune diseases. Up to now, numerous attempts at using Tregs to prevent or reverse T1D have been made [21]. Tregs, which have been defined by their prior expression of the forkhead winged helix transcription factor FoxP3, can prevent undesirable immune responses, such as the suppression of the auto-reaction of target $\mathrm{T}$ cells [23]. Importantly, SEB-T cells did not express FoxP3 in our study. Therefore, these cells cannot be regarded as Tregs even though they both prevent T1D through TGF- $\beta$ secretion [21].

Inflammatory cytokines (IFN- $\gamma$, IL-2 and IL-6) secretion and Th1 cells polarization can induce the occurrence of T1D in NOD mice [21]. In addition, numerous studies have indicated that anti-inflammatory cytokine (IL-4, IL-10) secretion and Th2 shift may be beneficial to T1D in NOD mice [8]. Unlike NKT cell therapy reported in literature, the serum level of cytokines secreted from Th1 and Th 2 cells did not appear polarized after infusion of SEB-T cells. We concluded that TGF- $\beta$ and not Th 2 cytokine secretion after infusion of SEB-T cells may be one of the factors ameliorating the hyperglycemia in the T1D NOD mice described above. IFN- $\gamma$ and IL- 2 secreted by activated effector $\mathrm{T}$ cells can result in enrichment of cytotoxic macrophages and $\mathrm{CD} 8^{+} \mathrm{T}$ cells, which infiltrate and destroy the pancreatic $\beta$ cells in NOD mice [21]. However, the expansion and function of Tregs requires the stimulatory signal IL-2 [24]. Interleukin 2 in combination with Tregs can maintain the self-tolerance in severe autoimmune disease that develops in mice deficient in IL-2 or IL-2R [25]. Furthermore, some studies have suggested that the IFN- $\gamma$ stimulated anti-diabetic environment in the pancreatic islets is protective against T1D [26]. Therefore, IL-2 and IFN- $\gamma$ are very beneficial for the treatment of T1D though they also may result in the occurrence of T1D.

Taken together, this is the first study to demonstrate that adoptive transfer of SEB-T cells can efficiently protect against T1D in NOD mice possibly via TGF- $\beta$ secretion. In this paper, we observed the general biological characteristics and protection against T1D of SEB-T cells. And these cells derived from lymphocytes with SEB stimulation enriched in $\mathrm{CD}^{+} \mathrm{NKT}$ cells and possessed immu- nomodulatory and TGF- $\beta$ secretion abilities. The results of animal experiments demonstrated that the infusion of these cells are not only well tolerated, but also can protect against T1D for NOD mice. In addition, TGF- $\beta$ was greatly secreted, as seen by serum analysis. These results demonstrate that the infusion of SEB-T cells enrichment of $\mathrm{CD} 8^{+} \mathrm{NKT}$ cells is a suitable therapy for T1D.

The authors thank for all of the animal feeders in Chinese People's Liberation Army General Hospital Animal Experiment Center for participating in the animal studies.

This work was supported in part by the National Natural Science Foundation of China (30671981 and 81072492) and the 863 Projects of Ministry of Science and Technology of China (2013AA020105 and 2012AA020502).

The authors declare no conflict of interest.

\section{References}

1. Stadinski B, Kappler J, Eisenbarth GS (2010): Molecular targeting of islet autoantigens. Immun 32: 446-456.

2. Willcox A, Richardson SJ, Bone AJ, et al. (2009): Analysis of islet inflammation in human type 1 diabetes. Clin Exp Immunol 155: 173-181.

3. Li CR, Baaten BJ, Bradley LM (2012): Harnessing memory adaptive regulatory $\mathrm{T}$ cells to control autoimmunity in type 1 diabetes. J Mol Cell Biol 4: 38-47.

4. Hong S, Wilson MT, Serizawa I, et al. (2001): The natural killer T-cell ligand alpha-galactosylceramide prevents autoimmune diabetes in non-obese diabetic mice. Nat Med 7: 1052-1056.

5. Sharif S, Arreaza GA, Zucker P, et al. (2001): Activation of natural killer $\mathrm{T}$ cells by alpha-galactosylceramide treatment prevents the onset and recurrence of autoimmune type 1 diabetes. Nat Med 7: 1057-1062.

6. Taniguchi M (1999): V alpha 14 NKT cells as a novel immune system. Nihon Rinsho Meneki Gakkai Kaishi 22: 363-367.

7. Seino K, Taniguchi M (2005): Functionally distinct NKT cell subsets and subtypes. J Exp Med 202: 1623.

8. Chen YG, Choisy-Rossi CM, Holl TM, et al. (2005): Activated NKT cells inhibit autoimmune diabetes through tolerogenic recruitment of dendritic cells to pancreatic lymph nodes. J Immunol 174: 1196-1204.

9. Naumov YN, Bahjat KS, Gausling R, et al. (2001): Activation of CD1d-restricted T cells protects NOD mice from developing diabetes by regulating dendritic cell subsets. Proc Natl Acad Sci U S A 98: 13838-13843.

10.Zhou D, Mattner J, Cantu C 3rd, et al. (2004): Lysosomal glycosphingolipid recognition by NKT cells. Science 306 : 1786-1789.

11. Kinjo Y, Wu D, Kim G, et al. (2005): Recognition of bacterial glycosphingolipids by natural killer T cells. Nature 434 : 520-525.

12.Chen G, Han G, Wang J, et al. (2008): Induction of active tolerance and involvement of CD1d-restricted natural killer $\mathrm{T}$ cells in anti-CD3 $\mathrm{F}\left(\mathrm{ab}\right.$ ') ${ }_{2}$ treatment-reversed new-onset diabetes in nonobese diabetic mice. Am J Pathol 172: 972-979. 
13. Kadri N, Korpos E, Gupta S, et al. (2012): CD4(+) type II NKT cells mediate ICOS and programmed death-1-dependent regulation of type 1 diabetes. J Immunol 188: 3138-3149.

14.D'Alise AM, Ergun A, Hill JA, et al. (2011): A cluster of coregulated genes determines TGF- $\{$ beta $\}$-induced regulatory T-cell (Treg) dysfunction in NOD mice. Proc Natl Acad Sci U S A 108: 8737-8742.

15.Jansen A, Homo-Delarche F, Hooijkaas H, et al. (1994): Immunohistochemical characterization of monocytes-macrophages and dendritic cells involved in the initiation of the insulitis and beta-cell destruction in NOD mice. Diabetes 43: 667-675.

16. Rajbhandary S, Zhao MF, Zhao N, et al. (2013): Multiple cytotoxic factors involved in IL-21 enhanced antitumor function of CIK cells Signaled through STAT-3 and STAT5b pathways. Asian Pac J Cancer Prev 14: 5825-5831.

17.Zorena K, Malinowska E, Raczyńska D, et al. (2013): Serum concentrations of transforming growth factor-Beta 1 in predicting the occurrence of diabetic retinopathy in juvenile patients with type 1 diabetes mellitus. J Diabetes Res 2013: 614908.

18. Ishigame H, Zenewicz LA, Sanjabi S, et al. (2013): Excessive Th1 responses due to the absence of TGF- $\beta$ signaling cause autoimmune diabetes and dysregulated Treg cell homeostasis. Proc Natl Acad Sci USA 110: 6961-6966.

19. Shull MM, Ormsby I, Kier AB, et al. (1992): Targeted disruption of the mouse transforming growth factor-beta 1 gene results in multifocal inflammatory disease. Nature 359: 693-699.

20.Fantini MC, Becker C, Monteleone G, et al. (2004): Cutting edge: TGF-beta induces a regulatory phenotype in $\mathrm{CD} 4{ }^{+} \mathrm{CD} 25^{-}$ $\mathrm{T}$ cells through Foxp3 induction and down-regulation of Smad7. J Immunol 172: 5149-5153.

21. Cabrera SM, Rigby MR, Mirmira RG (2012): Targeting regulatory $\mathrm{T}$ cells in the treatment of type 1 diabetes mellitus. Curr Mol Med 12: 1261-1272.

22. Thomas DC, Wong FS, Zaccone P, et al. (2013): Protection of islet grafts through TGF beta induced tolerogenic DC. Diabetes 62: 3132-3142.

23. Tang Q, Bluestone JA (2008): The Foxp3 $3^{+}$regulatory T cell: a jack of all trades, master of regulation. Nat Immunol 9: 239244.

24.Zhang H, Chua KS, Guimond M, et al. (2005): Lymphopenia and interleukin-2 therapy alter homeostasis of CD4+CD25+ regulatory T cells. Nat Med 11: 1238-1243.

25. Wolf M, Schimpl A, Hünig T (2001): Control of T cell hyperactivation in IL-2-deficient mice by $\mathrm{CD} 4(+) \mathrm{CD} 25(-)$ and CD4(+)CD25(+) T cells: evidence for two distinct regulatory mechanisms. Eur J Immunol 31: 1637-1645.

26. Duarte N, Stenström M, Campino S, et al. (2004): Prevention of diabetes in nonobese diabetic mice mediated by CD1d-restricted nonclassical NKT cells. J Immunol 173: 3112-3118. 